Revista de Derecho Politico, núm. 29, 1989, pp. 185-194

\title{
CRÓNICA PARLAMENTARIA DEL CONGRESO DE LOS DIPUTADOS
}

\author{
POR
}

PILAR MELLADO PRADO

La presente Crónica parlamentaria comprende la actividad desarrollada por la Cámara Baja durante el primer periodo de sesiones de 1988, en la III Legislatura constitucional.

\section{PRÁCTICAS APLICATIVAS DE LA CONSTITUCIÓN}

\section{De carácter no legislativo}

A) Informe del Defensor del Pueblo:

De acuerdo con lo dispuesto en la Ley Orgánica del Defensor del Pueblo, éste presentó a las Cortes su informe correspondiente a 1987 (E, 109), que fue aprobado por ambas Cámaras (DSC núm. 210, 15 de junio de 1988).

\section{De carácter legislativo}

\section{A) Convalidación de Decretos-leyes}

Durante este período de sesiones fueron convalidados los siguientes Decretos-leyes: 
RDL 1/1988, de 22 de febrero, por el

RDL 2/1988 de 15 de marzo, sobre

RDL 3/1988 de 3 de junio, por el que

Convalidado

se concede un suplemento de crédito al programa 513.D (creación de infraestructura de carretera).

\section{iI. ACTIVIDAD LEGISLATIVA}

A continuación se señalan los proyectos y proposiciones de ley que, a nuestro juicio, merecen una mayor atención.

\section{Proyectos de Ley}

De planta y organización territorial de la jurisdicción militar.

Mercado de valores.

Modificación de la Ley 30/84 de medidas para la Reforma de la Función Pública.
A, 72 Aprobado por Comisión con competencia legisla- tiva plena el 25-3-88.

A, 73 Aprobado el 20-7-88.

A, 74 Aprobado el 20-7-88. 


MATERIA BOCG INCIDENCIAS

Procesal Militar.

A, 79

Reforma parcial y adaptación de la Le-

A, 80 gislación mercantil a las Directivas de la CEE en materia de Sociedades.

Tasas y Precios Públicos.

A, 82

De demarcación y Planta Judicial.

A, 83

Regulación de las Haciendas Locales.

A, 85

Cooperativas de Crédito.

A, 86

Bases de Procedimiento Laboral

A, 88

Por el que se amplia a 16 semanas el permiso de maternidad.
A, 87

De la función estadistica pública.

A, 92

Conservación de los espacios naturales

A, 93 $y$ de la flora y fauna silvestres.

\section{Proposiciones de Ley}

MATERIA

Prevención de riesgos para la salud en el trabajo ante pantallas de datos.
B, 97 Rechazada el 27-9-88 C.D.S. 
Creación ael Fondo de Indemnizaciones derivadas de delitos comunes.

Modificación del artículo 30 de la LOTC.

Modificación del artículo 20 de la Ley de Enjuiciamiento Civil.

Modificación del artículo 563 bis b) del Código Penal.

Autorización al Gobierno a ampliar la actual duración máxima de los contratos temporales como medida de fomento al empleo.

Modificación de la pena impuesta por malos tratos a cónyuges o hijos menores comprendidos en el artículo 583 del Código Penal (Orgánica).

Ampliación de la licencia por embarazo de la mujer trabajadora.

Reforma del Reglamento del Congreso de los Diputados.

Por la que se regulan los derechos a la cláusula de conciencia y secreto profesional en el ejercicio de la libertad de información.

Contratos de la Administración Pública.
B, 99 Agrupación P.D.P.

Rechazada el 18-10-88.

B, 100

Agrupación

P.D.P.

B, 101

C. Popular

B, 102

C. Popular

B, 104

M. Catalana
B, 105

M. Catalana
B, 106

M. Catalana

B, 108

C. Popular

B, 109

C.D.S.

B, 110

C. Popular 
Por la que se reconocen los derechos de los trabajadores que adoptan un hijo.

Por la que se introduce un nuevo párrafo en el artículo 340 bis del Código Penal, relativo a los delitos contra la seguridad del tráfico (Orgánica).

Reguladora del Tráfico de influencias y del manejo de información privilegiada.

Supresión de la figura del Gobernador Civil y de reforma de la Ley que desarrolla el artículo 154 CE.

Modificación del artículo 16 de la Ley Reguladora del Registro Civil.

Modificación de los artículos 204 bis y 204 bis a) del Código Penal (Orgánica).

Protección de los derechos y libertades en relación con el uso de la informática y las telecomunicaciones (Orgánica).

Reforma del Reglamento del Congreso de los Diputados.
B, 113

M. Catalana

B, 114

C.D.S.
B, 115

C. Popular

B, 116

P.N.V.

B, 117

C. Popular

B, 118

IU-EC

B, 120

IU-EC

B, 122

Agrupación

Liberal 
III. ACTIVIDAD FINANCIERA

\section{Materia Presupuestaria}

\begin{tabular}{|c|c|c|}
\hline MATERIA & $B O C G$ & INCIDENCIAS \\
\hline $\begin{array}{l}\text { Concesión de Crédito Extraordinario } \\
\text { para completar abono Compañía Trans- } \\
\text { mediterránea de Bonificaciones de resi- } \\
\text { dentes en Ceuta, Canarias, Melilla y } \mathrm{Ba} \text { - } \\
\text { leares. }\end{array}$ & A, 75 & Aprobado el $13-6-88$ \\
\hline $\begin{array}{l}\text { Concesión de dos créditos extraordina- } \\
\text { rios de compensación de tasas universi- } \\
\text { tarias correspondientes a los cursos } 86 \text { / } \\
87 \text { y } 87 / 88 \text {. }\end{array}$ & A, 76 & Aprobado el 13-6-88 \\
\hline $\begin{array}{l}\text { Concesión de dos créditos para abonar } \\
\text { a la Sociedad Cooperativa de Viviendas } \\
\text { Sociales. }\end{array}$ & A, 78 & Aprobado el 13-6-88 \\
\hline $\begin{array}{l}\text { Concesión de dos créditos al Ministerio } \\
\text { de Educación y Ciencia, y al Ministerio } \\
\text { de Sanidad y Consumo para compensar } \\
\text { el déficit del Hospital Clínico y Provincial } \\
\text { de Barcelona. }\end{array}$ & A, 89 & Aprobado el $20-10-88$ \\
\hline
\end{tabular}

\section{Materia Tributaria}

MATERIA

Ampliación de las deducciones fiscales para los hijos menores de 6 años en el IRPF.
BOCG INCIDENCIAS

B, 103 


\section{ACTIVIDAD DE CONTROL SOBRE-EL GOBIERNO}

\section{Actos de control sin eficacia jurídica}

A continuación se señalan las proposiciones no de Ley e interpelaciones que se presentaron y/o debatieron es este período de sesiones, y que, a nuestro juicio, merecen una mayor atención.

\section{A) Proposiciones No de Ley (en Pleno)}

MATERIA

BOCG DSC

G. PROPONENTE

INCIDENCIAS
Sobre ampliación de la duración de determinadas prestaciones por desempleo.

Medidas de bonificación en las tarifas telefónicas de los jubilados.

Ratificación del Protocolo Adicional al Convenio Europeo de Derechos Humanos.

Para que se dicten normas reguladoras de la utilización por las autoridades politicas del Estado de los medios oficiales de transporte aéreo.

Nueva regulación de las familias numerosas a partir de los tres hijos.

Unión Europea y los poderes constituyentes del Parlamento Europeo.
D, 155
111
M. Catalana
Rechazada, D, 191
D, 155
134
M. Catalana
Aprobada, D, 227
D, 170108 Democracia Aprobada, D, 188 Cristiana
D, 176
115
C. Popular
Rechazada, D, 197
D, 183
$-$
M. Catalana

D, 197

124
IU-EC

Rechazada, D, 207 
B) Proposiciones No de Ley (en Comisión)

\begin{tabular}{|c|c|c|c|c|}
\hline MATERIA & $B O C G$ & $D S C$ & G. PROPONENTE & INCIDENCIAS \\
\hline $\begin{array}{l}\text { Contribución de España } \\
\text { a los planes defensivos } \\
\text { de la OTAN. }\end{array}$ & D, 149 & $\begin{array}{l}260 \\
\text { Defensa }\end{array}$ & P.D.P. & Aprobada, D, 179 \\
\hline $\begin{array}{l}\text { Discriminación de la mu- } \\
\text { jer inmigrante en la CEE. }\end{array}$ & $D, 149$ & 一 & P.D.P. & Retirada \\
\hline $\begin{array}{l}\text { Actualizacióin de las } \\
\text { cantidades asignadas a } \\
\text { la protección de la fami- } \\
\text { lia. }\end{array}$ & D, 157 & $\begin{array}{l}247 \\
\text { Política } \\
\text { Social y } \\
\text { Empleo }\end{array}$ & C.D.S. & Rechazada, D, 200 \\
\hline $\begin{array}{l}\text { Proceso de autodetermi- } \\
\text { nación del pueblo del } \\
\text { Sahara Occidental. }\end{array}$ & D, 179 & $\begin{array}{l}282 \\
\text { Asuntos } \\
\text { Exterio- } \\
\text { res }\end{array}$ & IU-Ec & Aprobada, D, 191 \\
\hline $\begin{array}{l}\text { Para fomentar el cine in- } \\
\text { fantil y juvenil. }\end{array}$ & D, 187 & $\begin{array}{l}300 \\
\text { Educa- } \\
\text { ción y } \\
\text { Cultura }\end{array}$ & M. Catalana & Aprobada, D, 200 \\
\hline $\begin{array}{l}\text { Incursión del Ejército is- } \\
\text { raelí en el Libano. }\end{array}$ & D, 207 & $\begin{array}{l}349 \\
\text { Asuntos } \\
\text { Exterio- } \\
\text { res }\end{array}$ & G. Socialista & Aprobada, D, 242 \\
\hline
\end{tabular}


C) Interpelaciones

C, 1) Ordinarias:

MATERIA BOCG DSC G. PROPONENTE INCIDENCIAS

Medidas de política general a adoptar por el

D, $108 \quad-\quad$ C. Popular Gobierno para mejorar el Servicio de Telefónica.

$C_{2}$ ) Urgentes

Deterioro del servicio de

D, $150 \quad 83 \quad$ P.D.P.

Correos.

Situación de las sectas en España.

D, 152

86

P.D.P.

Moción aprobada

Medidas que puede adoptar el Gobierno para me-
D, 152
86
C. Popular jorar la administración de Justicia en España.

Colocación ilegal de escuchas telefónicas.
D, 161
91
C. Popular

Sobre ilegalización de

D, 164

94

C. Popular Herri Barasuna.

Huelga del profesorado

D, 164

94

C.D.S. no universitario.

Sobre fusiones banca- $\quad$ D, $203 \quad 122 \quad$ G. Mixto rias. 


MATERIA BOCG DSC G. PROPONENTE INCIDENCIAS

Situación del Empleo ju-
D, 208
125
IU-EC venil.

Sobre «Spantax».

D, $208 \quad 125$

Demo-

cracia

Cristiana

Funcionamiento de Correos y Teléfonos y su inciden-
D, 209
128
C.D.S.

cia en la política general.
Política general de
D, 209
128
C. Popular.

Carreteras.

\section{ACTIVIDAD EN RELACIÓN CON LAS COMUNIDADES AUTONOMAS}

\section{Actividad de carácter normativo}

MATERIA BOCG INCIDENCIAS

Transferencia de competencias en materia de educación a la Comunidad Autónoma de Castilla-León (Orgánica).

B, 119

Cortes de CastillaLeón. 